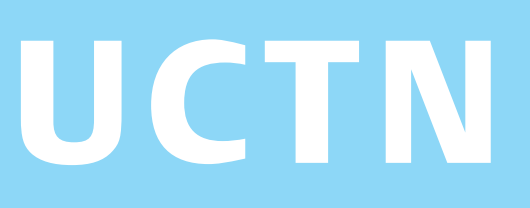

\title{
Is the fluoroscopic image always a true represen- tation of the position of an endoscopically placed device?
}

Malignant lesions are the most common cause of large-bowel obstruction, accounting for more than 150000 deaths annually worldwide [1], and up to $75 \%$ of colorectal cancers occur in the left colon [2]. Since their first description by Dohmoto in 1991 [3], self-expandable metal stents (SEMS) have provided an alternative to surgery for effective palliation in the management of colorectal obstructions [4]. Despite the fact that the technique of endoscopic SEMS placement under radiographic control has become fairly standard, the fluoroscopic image of the guide wire correctly positioned through a malignant stricture might sometimes not be a true representation of the actual position, however.

We report the case of a 55-year-old woman with silent pathological remote anamnesis, who had a sigmoid adenocarcinoma. In her case, the radiographic image seemed to show correct placement of the guide wire inside the proximal bowel, over the malignant stricture, with regular filling, even though the wire had in fact passed through the neoplasm and out of the bowel altogether instead of into the residual lumen (surgical finding). This had produced an image in which a filling of the abdominal cavity looked very similar to an image of a contrast-filled bowel. Stent opening (Wallstent $9 \mathrm{~cm} \times 22 \mathrm{~mm}$; Microvasive Endoscopy, Boston Scientific Corp., Natick, Massachusetts, USA.) then caused a bowel perforation (Figure 1-4) and the patient had to undergo a left hemicolectomy. It is therefore very important to be sure of correct filling of the proximal bowel before SEMS placement, paying particular attention to evaluation of the contrast fluoroscopic image over the stricture.

Endoscopy_UCTN_Code_CPL_1AJ_2AF

\section{B. Mangiavillano, E. Masci, P. A. Testoni} Division of Gastroenterology and Gastrointestinal Endoscopy, University Vita-Salute San Raffaele, Scientific Institute San Raffaele, Milan, Italy.

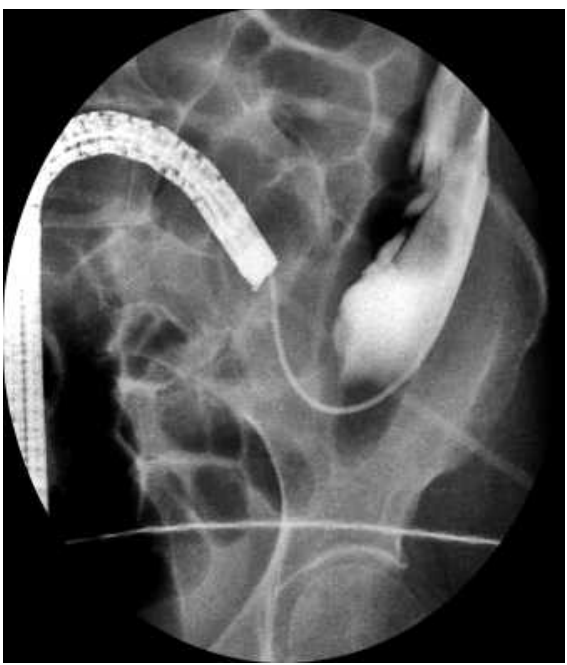

Figure 1 Radiographic image showing contrast agent outside the bowel before placement of the self-expandable metallic stent (SEMS).

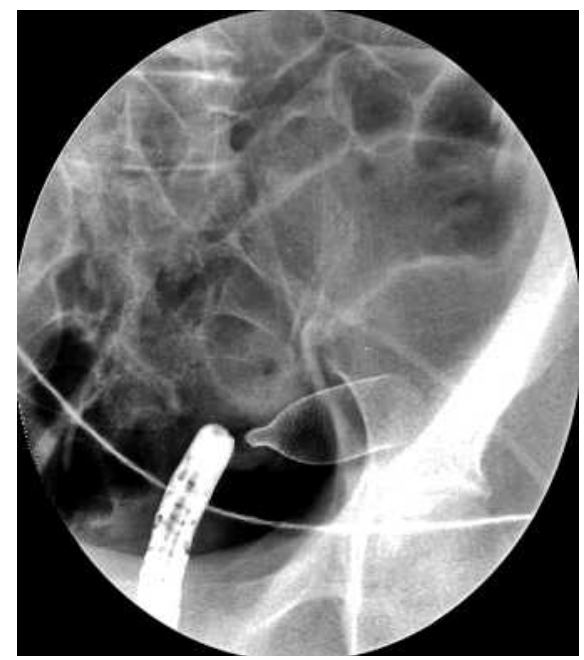

Figure 2 The distal end of the SEMS has not expanded because it is inside the neoplasm.

\section{References}

${ }^{1}$ World Health Organization. World Health Statistics Annual.Geneva: World Health Organization 1989

2 Deans GT, Krukowski ZH, Irwin ST. Malignant obstruction of the left colon. Br J Surg 2002; 89: 1069-1102

${ }^{3}$ Dohmoto M. New method: endoscopic implantation of rectal stent in palliative treatment of malignant stenosis. Endosc Dig 1991; 3: 1507-1512

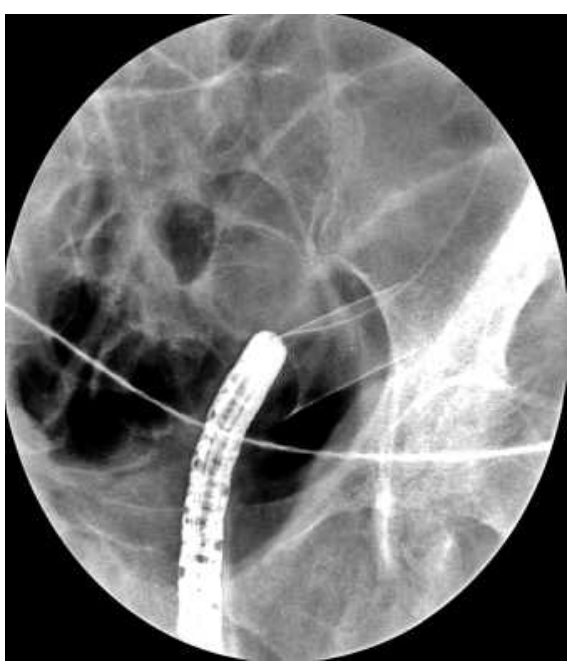

Figure 3 The SEMS, totally expanded, outside the bowel.

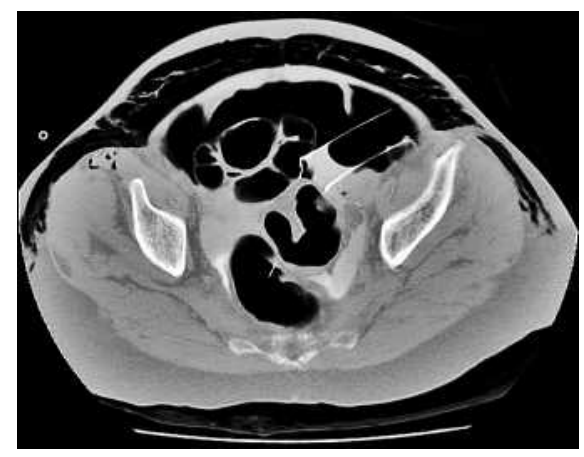

Figure 4 Computed tomographic image showing the expanded stent outside the colonic lumen, in the left iliac region.

${ }^{4}$ Bhardwaj R, Parker MC. Palliative therapy of colorectal carcinoma: stent or surgery? Colorectal Dis 2003; 5: 518-521

\section{Corresponding Author}

\section{B. Mangiavillano, M.D.}

Division of Gastroenterology and

Gastrointestinal Endoscopy

University Vita-Salute San Raffaele

Scientific Institute San Raffaele

Via Olgettina 62

20132 Milan

Italy

Fax: $\quad$ Fax: +39-02-26432504

E-mail: mangiavillano.benedetto@hsr.it

DOI: 10.1055/s-2006-944705 